\title{
Microcephaly and developmental delay caused by short-chain acyl-CoA dehydrogenase deficiency
}

\author{
Mustafa Kılıç ${ }^{1}$, Saliha Şenel ${ }^{2}$, Kadri Karaer ${ }^{3}$, Serdar Ceylaner ${ }^{3}$ \\ ${ }^{1}$ Division of Metabolism, ${ }^{2}$ Department of Pediatrics, Dr. Sami Ulus Children Hospital, ${ }^{3}$ Intergen Laboratory, Ankara, \\ Turkey. E-mail: kilickorkmaz@yahoo.com.tr \\ Received: 16th February 2017, Revised: 18th April 2017, Accepted: 23th April 2017
}

SUMMARY: Kılıç M, Şenel S, Karaer K, Ceylaner S. Microcephaly and developmental delay caused by short-chain acyl-CoA dehydrogenase deficiency. Turk J Pediatr 2017; 59: 708-710.

We report a four-year-old girl who presented with intrauterine growth retardation, mild dysmorphism, cleft palate, microcephaly, developmental delay, epilepsy and recurrent lower respiratory tract infection and diagnosed shortchain acyl-CoA dehydrogenase deficiency. Metabolic evaluation and molecular analysis confirmed the diagnosis. In spite of many patients already known in literature, this is one of the rarest reports of a Turkish patient. This suggests selective metabolic screening should be done in every patient with unknown etiology of neurological disorder. Furthermore, newborn screening using tandem mass spectrometry may prevent this severe neurological impairment.

Key words: ACADS, SCAD, microcephaly, developmental delay, epilepsy.

Short-chain acyl-CoA dehydrogenase (SCAD, OMIM 606885) deficiency (SCADD, OMIM 201470) is a rare autosomal recessive inherited disorder of mitochondrial fatty acid oxidation defect encoded by ACADS gene located on chromosome $12 \mathrm{q} 24$. It has variable clinical phenotypes ranging from asymptomatic to severe neurological presentation. ${ }^{1-6}$ Birth prevalence of SCADD from newborn screening was reported to be approximately 1 in $35,000.5$ Because the enzyme is a flavoprotein, riboflavin therapy is one of the treatment options who have relatively low FAD levels. ${ }^{7,8}$ Low fat diet, frequently feeding and carnitine supplementation are also suggested. We report a four-year-old girl with severe neurological abnormalities suffering from SCADD.

\section{Case Report}

Four-year-old female Turkish patient was admitted to hospital with the complaint of cough, wheezing and respiratory distress. From the history, it was learned that she was operated for cleft palate at 10-months of age, had recurrent hospitalization due to lower respiratory tract infection and had been on $\mathrm{Na}$-valproate $20 \mathrm{mg} / \mathrm{kg} /$ day therapy for epilepsy after age three. She could not walk alone or speak fluently. She was delivered spontaneously at term with $2,000 \mathrm{~g}$ birth weight as a third child of healthy parents after uneventful pregnancy and birth. Parents were second degree cousins. There was no family history of similar disease. Physical examination revealed microcephaly $(41 \mathrm{~cm}$; < $-2 \mathrm{SD})$, mild dysmorphism, scar tissue in soft palate, developmental delay, failure to thrive (weight: $11 \mathrm{~kg},<-2 \mathrm{SD}$; height: $88 \mathrm{~cm},<-2$ SD; weight for height was $80-90 \%]$, bilateral crackles at the bottom of lungs and increased expirium time. She could speak only single words and walk with help. Muscle tone was decreased slightly in lower extremities. Deep tendon reflexes were normal and Babinsky sign was bilaterally flexor. Eye examination was normal. She was hospitalized for the treatment of lower respiratory tract infection and further evaluation. Antibiotic therapy was started. Laboratory investigation including blood count, liver transaminases and kidney function tests, venous blood gases, immunoglobulins, sweet chloride test, creatine kinase, cholesterol, thyroid hormones, TORCH serology, lactate, pyruvate, ammonia, quantitative plasma and urine aminoacid analyses, phenylalanine level of the mother and chromosome analyses were 
normal. C-reactive protein was increased. Direct X-ray of skeletal system showed microcephaly and bilaterally coxa valgus deformities. Echocardiography showed secundum atrial septal defect (ASD). Electroencephalography was normal. Brain magnetic resonance imaging (MRI) demonstrated microcephaly and arachnoid cyst. Developmental test with Bayley II pointed that she was nearly one-year-old for motor and cognitive field. Metabolic investigations by tandem mass spectrometry showed elevated level of C4carnitine (butyrylcarnitine) $(2.32 \mu \mathrm{mol} / \mathrm{L}$, $\mathrm{N}<1.1$ ) in plasma and urine organic acid analyses revealed elevated level of ethylmalonic acid excretion $(720 \mathrm{mmol} / \mathrm{mol}$ creatinine, $\mathrm{N}<18$ ) which suggested the diagnosis of patient with SCADD. Direct sequencing of ACADS gene showed c.1147C>T;p.R383C homozygous mutation and confirmed the diagnosis. In addition to antiepileptic drug, L-carnitine $50 \mathrm{mg} / \mathrm{kg} /$ day, riboflavin $10 \mathrm{mg} /$ $\mathrm{kg} /$ day treatment and frequently feeding with low fat diet were recommended. We have not observed hypoglycemia and seizures on follow up for six months. It was not expected to get full recovery due to late diagnosis with this severely affected patient, however it needs time to see the exact benefits of these therapies.

Written consent of the parents was obtained for the publication.

\section{Discussion}

SCADD, was first described in 1987 and thereafter the responsible gene was identified in $1990 .{ }^{9,10}$ To date, nearly 70 mutations and two common variants, c.511C $>\mathrm{T}$ and c. $625 \mathrm{G}>\mathrm{A}$ have been reported in the ACADS gene. $1,3,5,6,11$ Genotype-phenotype correlation is weak and poorly understood. ${ }^{6}$ Although these two common variants can be seen in general populations (mostly asymptomatic), it can also present with severe neurological abnormalities. Environmental (fever, infection, metabolic stress, starvation, hypoglycemia, etc.) and genetic (modifier genes) factors may be responsible for clinical and genetic variability. Therefore, SCADD is considered as a protein misfolding disorder and can lead to clinical disorder in combination with other genetic and environmental factors. ${ }^{11}$ In our observation from the presented Turkish patient and the reported clinical cases, patients with higher ethylmalonic acid (EMA) levels had more severe clinical condition. We agree that it might be explained with the view that EMA is related to mitochondrial dysfunction and oxidative stress. ${ }^{11-14}$

The clinical spectrum of SCADD is extremely heterogeneous. It can vary from asymptomatic cases to feeding problems, ketotic hypoglycemia, metabolic acidosis, lethargy, weakness, hypotonia, microcephaly, developmental delay, speech delay, behavioral disturbances, epilepsy, myopathy, neuropathy, dysmorphic features, optic atrophy. ${ }^{1-5}$ SCADD is generally diagnosed as a result of investigation of neurological disorders and/or hypoglycemia by selective screening. In recent years, newborn screening by tandem mass spectrometry has led to identification of newborns, mostly asymptomatic. ${ }^{3,5}$

At first we thought CNS (central nervous system) developmental anomalies, TORCHs infections, genetic and metabolic disorders for the differential diagnosis. Maternal phenylketonuria syndrome, serine synthesis defects, GLUT 1 deficiency, molybdenum cofactor deficiency, sulphite oxidase defect and Smith-Lemli Opitz syndrome are some of the metabolic disorders in the list of microcephaly. While we were investigating, most important clues were obtained from the metabolic screening results for the correct diagnosis. Elevated levels of C4-carnitine (butyrylcarnitine) in plasma and excretion of EMA in urine suggested SCADD in this patient. Molecular analyses confirmed the exact diagnosis. This mutation c.1147C > T;p.R383C had been previously described in homozygous state with a homozygous c. $625 \mathrm{G}>\mathrm{A}$ variant without any clinical signs. ${ }^{6}$ Interestingly, in the same study one patient with different mutations (c.529T >C; c.625G >A) had the same clinical signs as our patient with ASD, cleft lip/palate, and speech delay. That patient also had a deletion 22q11 region and was diagnosed with velocardiofacial syndrome as a second disease. We also planned to make FISH analyses for the second disease in our patient.

In spite of many patients already known in literature, only a few Turkish patients were reported. ${ }^{15.16}$ Bok et al. ${ }^{15}$ reported a homozygous c.1138C $>$ T mutation in two Turkish siblings, 
one had transient cholestasis, maternal HELLP syndrome, premature delivery but normal mental development, slight hypotonia, active behavior at three-year-old while the other sibling was asymptomatic. Kiykim et al. ${ }^{16}$ reported a homozygous c.625G $>$ A mutation in a Turkish patient with delayed speech, epilepsy and behavioral disturbances.

We suggest selective screening should be done in every patient with unknown neurological disorder. Furthermore, newborn screening may help us for early diagnosis, carefully follow-up and treatment of these patients to prevent disease complications.

\section{Acknowledgements}

We thank to the family of the patient for their participation involving informed consent.

\section{REFERENCES}

1. Corydon MJ, Vockley J, Rinaldo P, et al. Role of common gene variations in the molecular pathogenesis of shortchain acyl-CoA dehydrogenase deficiency. Pediatr Res 2001; 49: 18-23.

2. Tein I, Elpeleg O, Ben-Zeev B, et al. Short-chain acyl-CoA dehydrogenase gene mutation (c.319C $>\mathrm{T}$ ) presents with clinical heterogeneity and is candidate founder mutation in individuals of Ashkenazi Jewish origin. Mol Genet Metab 2008; 93: 179-189.

3. Waisbren SE, Levy HL, Noble M, et al. Short-chain acylCoA dehydrogenase (SCAD) deficiency: An examination of the medical and neurodevelopmental characteristics of 14 cases identified through newborn screening or clinical symptoms. Mol Genet Metab 2008; 95: 39-45.

4. van Maldegem BT, Wanders RJ, Wijburg FA. Clinical aspects of short-chain acyl-CoA dehydrogenase deficiency. J Inherit Metab Dis 2010; 33: 507-511.

5. Gallant NM, Leydiker $\mathrm{K}$, Tang $\mathrm{H}$, et al. Biochemical, molecular, and clinical characteristics of children with short chain acyl-CoA dehydrogenase deficiency detected by newborn screening in California. Mol Genet Metab 2012; 106: 55-61.

6. Pena L, Angle B, Burton B, Charrow J. Follow-up of patients with short-chain acyl-CoA dehydrogenase and isobutyryl-CoA dehydrogenase deficiencies identified through newborn screening: One center's experience. Genet Med 2012; 14: 342-347.
7. van Maldegem BT, Duran M, Wanders RJ, Waterham HR, Wijburg FA. Flavin adenine dinucleotide status and the effects of high-dose riboflavin treatment in short-chain acyl-CoA dehydrogenase deficiency. Pediatr Res 2010; 67: 304-308.

8. Lucas TG, Henriques BJ, Rodrigues JV, Bross P, Gregersen N, Gomes CM. Cofactors and metabolites as potential stabilizers of mitochondrial acyl-CoA dehydrogenases. Biochim Biophys Acta 2011; 1812: 1658-1663.

9. Amendt BA, Greene C, Sweetman L, et al. Short-chain acyl-coenzyme A dehydrogenase deficiency. Clinical and biochemical studies in two patients. J Clin Invest 1987; 79: 1303-1309.

10. Naito E, Indo Y, Tanaka K. Identification of two variant short chain acyl-coenzyme A dehydrogenase alleles, each containing a different point mutation in a patient with short chain acyl-coenzyme a dehydrogenase deficiency. J Clin Invest 1990; 85: 1575-1582.

11. Pedersen CB, Kølvraa S, Kølvraa A, et al. The ACADS gene variation spectrum in 114 patients with shortchain acyl-CoA dehydrogenase (SCAD) deficiency is dominated by missense variations leading to protein misfolding at the cellular level. Hum Genet 2008; 124: 43-56.

12. Pedersen CB, Zolkipli Z, Vang S, et al. Antioxidant dysfunction: potential risk for neurotoxicity in ethylmalonic aciduria. J Inherit Metab Dis 2010; 33: 211-222.

13. Schmidt SP, Corydon TJ, Pedersen CB, Bross $P$, Gregersen N. Misfolding of short-chain acyl-CoA dehydrogenase leads to mitochondrial fission and oxidative stress. Mol Genet Metab 2010; 100: 155-162.

14. Amaral AU, Cecatto C, Busanello EN, et al. Ethylmalonic acid impairs brain mitochondrial succinate and malate transport. Mol Genet Metab 2012; 105: 84-90.

15. Bok LA, Vreken P, Wijburg FA, et al. Short-chain AcylCoA dehydrogenase deficiency: studies in a large family adding to the complexity of the disorder. Pediatrics 2003; 112: 1152-1155.

16. Kiykim E, Zeybek CA, Zubarioglu T, et al. Inherited metabolic disorders in Turkish patients with autism spectrum disorders. Autism Res 2016; 9: 217-223. 